\title{
Defining the expression and metabolic regulation of alternative isoforms of the lipodystrophy protein seipin in developing adipocytes
}

\author{
E. Persiani and J.J. Rochford \\ Rowett Institute, School of Medicine and Dentistry, University of Aberdeen, Foresterhill campus, \\ Aberdeen AB25 2ZD, UK.
}

Disruption of BSCL2 gene, encoding the protein seipin, causes Congenital Generalized Lipodystrophy (CGL), the most severe lipodystrophy. Seipin is an endoplasmic reticulum membrane protein and has a fundamental role in adipose tissue development and maintenance, as demonstrated by cellular and in-vivo models ${ }^{(1-2)}$.

We have recently shown that seipin forms highly ordered dodecamers that creates a scaffold for critical pro-adipogenic enzymes (lipin-1 and 1-acylglycerol-3-phosphate-O-acyltransferase 2, AGPAT2) to act in a single functional complex ${ }^{(3)}$. Co-immunoprecipitation and immunofluorescence experiments in overexpressing HEK293 cells led us to extend these findings to show that seipin also binds 1-acylglycerol-3-phosphate-O-acyltransferase 1 (AGPAT1, Figure 1), glycerol-3-phosphate acyltransferase 3 (GPAT3) and glycerol-3phosphate acyltransferase 4 (GPAT4), implying an effect in lipid metabolism.

Sequences annotated in ENSEMBL suggest that several splice variants of seipin may exist. We focused our study on alternative splicing of exon 2 of seipin which generates either the 462 amino acid form of seipin (seipin-L) or the short 398 amino acid form of the protein (seipin-S). Gene expression profiles and proteomic analysis revealed that mature adipocytes mainly express seipin-S and that different isoforms have a distinct tissue-specific regulation (Figure 2) which is nutritionally controlled by dietary fat intake (data not shown). We also detected with qPCR analysis the skipping of exon 8 of seipin (seipin- $\Delta x 8$ ) in adipocyte cell lines, which would give rise to an isoform of seipin lacking key functional domains. We used this cloned spliced variant to investigate the ability to interact with binding partners and hypothesise the molecular significance of this novel transcripts by performing co-immunoprecipitation experiment in HEK293 cells (data not shown).
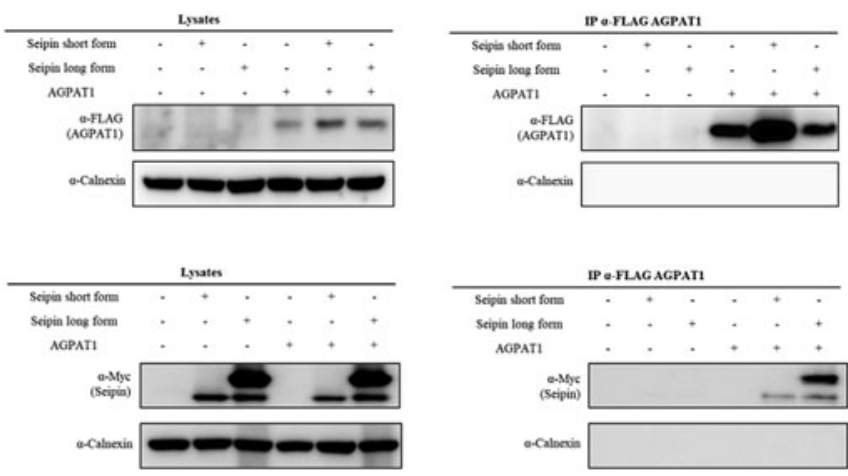

Fig. 1.
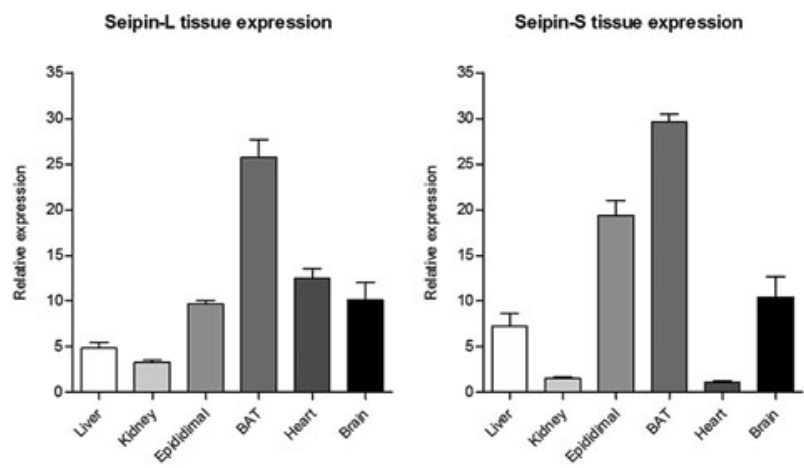

Fig. 2.

In conclusion, we extended the seipin binding partners to AGPAT1, GPAT3 and GPAT4 further supporting the model in which seipin acts as a molecular scaffold. These interactions may be fundamental in the regulation of lipid handling and the coordinated generation of pro-adipogenic signals. In addition, we have identified alternative seipin splicing events, which give rise to unrelated polypeptide sequences and could alter the ability to bind interacting partners. Overall this work reveals novel insights regarding seipin, a critical regulator of human adipose development and lipid metabolism, including its regulation by dietary lipids.

1. Payne VA, Grimsey N, Tuthill A, Virtue S, Gray SL, et al. (2008) Diabetes 57, 2055-2060.

2. Cui X, Wang Y, Tang Y, et al. (2011) Hum Mol Genet 20, 3022-3030.

3. Talukder MMU, Sim MFM, et al. (2015) Mol Metab 4, 199-209. 